\title{
Variation in the Integration Site for Capsule Gene Cluster in the Genome among Strains of Lactococcus garvieae
}

\author{
Kinya Kanai ${ }^{1 *}$, Toshio Honma ${ }^{2}$, Akane Souda $^{2}$, Kimihiro Shutou $^{2}$ and Yukitaka Sugihara ${ }^{3}$ \\ ${ }^{1}$ Graduate School of Fisheries and Environmental Sciences, \\ Nagasaki University, Nagasaki 852-8521, Japan \\ ${ }^{2}$ Bio Science Co, Ltd, Tokushima 779-1292, Japan \\ ${ }^{3}$ Nagasaki Prefectural Institute of Fisheries, \\ Nagasaki 851-2213, Japan
}

(Received October 9, 2017)

\begin{abstract}
Lactococcus garvieae is known as a pathogen of freshwater and marine fish species worldwide. L. garvieae isolates of serotype I have been divided into two serological phenotypes, namely $\mathrm{KG}^{-}$and $\mathrm{KG}^{+}$, which are differentiated by the presence or absence of polysaccharide capsule, and a phenotypic change from $\mathrm{KG}^{-}$to $\mathrm{KG}^{+}$occurs during repeated subculturing. When we subcultured laboratory collections of $L$. garvieae $\mathrm{KG}^{-}$strains repeatedly, they were divided into two groups. One group consisted of the strains which changed phenotypically after a relatively small number of times of subculturing, and the other group consisted of those which hardly changed. Genetic analyses revealed that the capsule gene cluster of the strains in the former group was integrated in a plasmid (CPS plasmid) and that in the latter group it was integrated in the chromosome or in both CPS plasmid and chromosome simultaneously. In the present study, we found three novel CPS plasmids. These plasmids were similar in structure to the CPS plasmid pBSLG13015 which was previously found in L. garvieae filefish isolates. In addition, we found a novel integration site of chromosomal capsule gene cluster. The majority of strains isolated before 1991 were those whose capsule gene cluster was integrated in the CPS plasmids.
\end{abstract}

Key words: Lactococcus garvieae, capsule gene cluster, $\mathrm{CPS}$ plasmid, $\mathrm{KG}^{-}, \mathrm{KG}^{+}$, phenotypic change

Lactococcus garvieae is known as a fish pathogen causing damages in aquaculture industry worldwide. In Japan lactococcosis occurs frequently among Seriola species, but other marine fish species including Japanese flounder Paralichthys olivaceus, Pacific bluefin tuna Thunnus orientalis and filefish, thread-sail filefish Stephanolepis cirrhifer and black scraper Thamnaconus modestus, are also susceptible to the disease (Kawanishi et al., 2006; Minami et al., 2012; Yoshida, 2016). L. garvieae isolates of serotype I have been divided into two serological phenotypes, namely $\mathrm{KG}^{-}$and $\mathrm{KG}^{+}$types, which are differentiated by an agglutination test using $\mathrm{KG}^{-}$and $\mathrm{KG}^{+}$antisera (Kitao, 1982). The $\mathrm{KG}^{-}$type produces a capsule on its cell-surface, is pathogenic to fish and possesses a gene cluster for capsular polysaccharide biosynthesis (capsule gene cluster) in the chromosome (Yoshida et al., 1997; Miyauchi et al., 2012). It was reported that the change from $\mathrm{KG}^{-}$to $\mathrm{KG}^{+}$type occurred during repeated subculturing (Kitao, 1982), and it is speculated that the capsule gene cluster of $\mathrm{KG}^{-}$type

\footnotetext{
* Corresponding author

E-mail: kanai@nagasaki-u.ac.jp
}

is lost during subculturing (Miyauchi et al., 2012).

Recently we found that the phenotypic change from $\mathrm{KG}^{-}$to $\mathrm{KG}^{+}$occurred more easily in $L$. garvieae isolates derived from filefish than those from other fish species, and reported that all the tested filefish $\mathrm{KG}^{-}$strains possessed a plasmid carrying the capsule gene cluster (CPS plasmid), which we named pBSLG13015 (Kanai et al., 2017). This plasmid was not detected in $\mathrm{KG}^{+}$derivatives of filefish $\mathrm{KG}^{-}$strains. Therefore, it was considered that the phenotypic change in filefish isolates was attributed to the loss of the CPS plasmid.

Approximately two decades ago we noticed that there were two groups in stocked $L$. garvieae $\mathrm{KG}^{-}$strains derived from yellowtail Seriola quinqueradiata. One group consisted of the strains in which the phenotypic change occurred after a relatively small number of times of subculturing, and the other group consisted of those in which the phenotypic change hardly occurred (Fig. 1). At that time, we could not clarify the reason for the presence of these two groups, but, as mentioned above, the fact that $L$. garvieae strains from filefish possessed the CPS plasmid promoted us to investigate the presence or absence of CPS plasmids in laboratory-stocked 


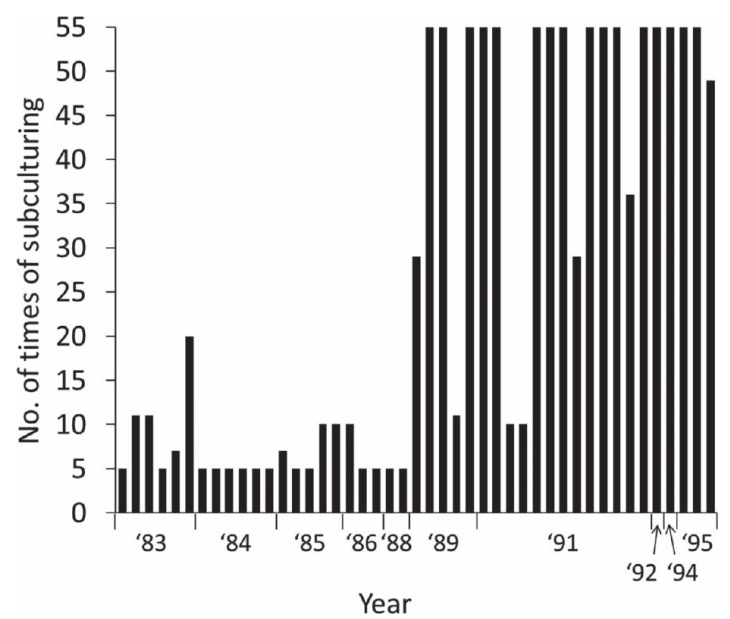

Fig. 1. Phenotypic change occurred in $L$. garvieae $\mathrm{KG}^{-}$strains isolated between 1983 and 1995 by repeated subculturing on Todd Hewitt agar. Each bar represents the number of times of subculturing that caused a change to $\mathrm{KG}^{+}$phenotype in each strain. The bars reached 55 mean that those strains did not change to $\mathrm{KG}^{+}$by 55 times of subculturing.

L. garvieae serotype I strains derived from diseased marine fish species other than filefish. In addition, at that time we also noticed that antibiotic susceptibility was different between the two groups, that is, strains of the former group were susceptible to erythromycin, but more than half of those of the latter group were resistant to this antibiotic. Thus, in the present study relationship between the integration site of the capsule gene cluster and antibiotic susceptibility of the stocked $L$. garvieae strains was also investigated.

\section{Materials and Methods}

\section{Bacterial strains}

A total of 152 L. garvieae strains isolated from diseased marine fish at aquaculture sites in western Japan were used in this study (Table 1). From these strains, NUF18, NUF462, Ns12sousui23 and Ns15sousui29 were chosen for genetic analysis as representative strains (Table 2). The serological phenotype of the 152 strains was determined by a slide agglutination test using $\mathrm{KG}^{-}$and $\mathrm{KG}^{+}$rabbit antisera (Kanai et al., 2017). Before the test, each strain was divided by colony morphology (see Results). The strains were cultured on Todd Hewitt (TH) agar (Difco) at $27^{\circ} \mathrm{C}$ and stocked by freezing at $-80^{\circ} \mathrm{C}$ in a deep freezer or at $-196^{\circ} \mathrm{C}$ in liquid nitrogen.

\section{Detection of capsule gene cluster}

The presence or absence of $L$. garvieae capsule gene cluster in the $\mathrm{KG}^{-}$and/or $\mathrm{KG}^{+}$phenotype of each strain was examined by the PCR method described previously (Kanai et al., 2017) with some modifications. Primer pairs used were Lg-Cps-38F/Lg-Cps-36R for the
Table 1. Source of Lactococcus garvieae strains used in this study

\begin{tabular}{|c|c|c|c|c|c|c|c|c|}
\hline \multirow[b]{3}{*}{ Year } & \multicolumn{8}{|c|}{ No. of strains } \\
\hline & \multicolumn{3}{|c|}{ Seriola } & \multirow[b]{2}{*}{ 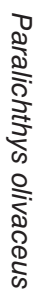 } & \multirow[b]{2}{*}{ 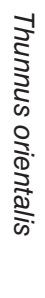 } & \multirow[b]{2}{*}{ 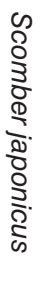 } & \multirow[b]{2}{*}{ 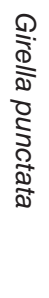 } & \multirow[b]{2}{*}{ Total } \\
\hline & 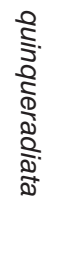 & 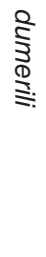 & 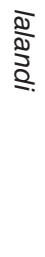 & & & & & \\
\hline 1983 & 10 & & & & & & & 10 \\
\hline 1984 & 7 & & & & & & & 7 \\
\hline 1985 & 15 & & & & & & & 15 \\
\hline 1986 & 8 & & & & & & & 8 \\
\hline 1987 & 8 & & & & & & & 8 \\
\hline 1988 & 11 & & & & & & & 11 \\
\hline 1989 & 25 & & & & & & & 25 \\
\hline 1991 & 19 & & & & & & & 19 \\
\hline 1992 & 5 & & & & & & & 5 \\
\hline 1993 & & & & 1 & & & & 1 \\
\hline 1994 & 1 & & & & & & & 1 \\
\hline 1995 & 3 & & & & & & & 3 \\
\hline 2002 & & & & 4 & & & & 4 \\
\hline 2005 & & 1 & & & & & & 1 \\
\hline 2006 & 1 & & & & & & & 1 \\
\hline 2007 & 2 & & & & & 1 & & 3 \\
\hline 2008 & 2 & & 1 & & & & & 3 \\
\hline 2009 & 3 & 1 & & & & & & 4 \\
\hline 2011 & 1 & 2 & 3 & & & & 1 & 7 \\
\hline 2012 & & 2 & & 1 & 2 & & & 5 \\
\hline 2013 & & & 2 & & 1 & & & 3 \\
\hline 2014 & & & & & 1 & & & 1 \\
\hline 2015 & 2 & & 1 & & & & & 3 \\
\hline 2016 & 1 & & 2 & & 1 & & & 4 \\
\hline Total & 124 & 6 & 9 & 6 & 5 & 1 & 1 & 152 \\
\hline
\end{tabular}

chromosomal gene cluster designed from L. garvieae Lg2 complete genome sequence (GenBank accession no. NC_017490) and Lg-pF2/Lg-pR2 for the plasmid gene cluster (Table 3). PCR was performed on C1000 Thermal Cycler (Bio-Rad) using KOD FX Neo Polymerase (Toyobo). The PCR reaction mixture $(50 \mu \mathrm{L})$ contained $25 \mu \mathrm{L}$ of $2 \times$ PCR buffer for KOD FX Neo (Toyobo), $0.4 \mathrm{~mm}$ of each dNTP, $0.3 \mu \mathrm{m}$ of each primer, $1 \mu \mathrm{L}$ of template DNA and $0.5 \mathrm{U}$ of KOD FX Neo Polymerase. The PCR condition was as follows; initial denaturation at $94^{\circ} \mathrm{C}$ for 2 min and 30 cycles of denaturation at $98^{\circ} \mathrm{C}$ for $10 \mathrm{~s}$, annealing at $60^{\circ} \mathrm{C}$ for $30 \mathrm{~s}$ and extension at $68^{\circ} \mathrm{C}$ for $9 \mathrm{~min}$. The PCR products were electrophoresed in $0.8 \%$ agarose gel with Quick Load 1 kb Extend DNA Ladder (New England BioLabs) and stained with ethidium bromide.

Nucleotide sequence analysis of integration site of capsule gene cluster

The genomic DNAs of $L$. garvieae NUF18, NUF462, Ns12sousui23 and Ns15sousui29 were isolated using Wizard Genomic DNA Purification kit (Promega) 
Table 2. Representative L. garvieae strains used for genetic analysis

\begin{tabular}{|c|c|c|c|c|c|c|}
\hline \multirow{2}{*}{ Strain } & \multicolumn{3}{|c|}{ Source } & \multirow{2}{*}{ Phenotype } & \multirow[b]{2}{*}{ CPS plasmid } & \multirow{2}{*}{$\begin{array}{c}\text { Chromosomal } \\
\text { capsule gene } \\
\text { cluster }\end{array}$} \\
\hline & Fish & Prefecture & Year & & & \\
\hline NUF18 & Yellowtail & Nagasaki & 1983 & $\mathrm{KG}^{-}$ & + & - \\
\hline NUF462 & Yellowtail & Kumamoto & 1989 & $\mathrm{KG}^{-}$ & + & + \\
\hline Ns12sousui23 & Pacific bluefin tuna & Nagasaki & 2012 & $\mathrm{KG}^{-}$ & + & - \\
\hline Ns15sousui29 & Kingfish & Nagasaki & 2015 & $\mathrm{KG}^{-}$ & + & - \\
\hline
\end{tabular}

Yellowtail, Seriola quinqueradiata; kingfish, S. Ialandi; Pacific bluefin tuna, Thunnus orientalis.

Table 3. PCR primers used for the detection of capsule gene cluster in CPS plasmids and chromosomes of $L$. garvieae

\begin{tabular}{lll}
\hline \multicolumn{1}{c}{ Primer* } & \multicolumn{1}{c}{ Sequence $\left(5^{\prime}-3^{\prime}\right)$} & \multicolumn{1}{c}{ Target } \\
\hline Lg-pF2 & GTGCCATAAACATCGTCTGGTTG & CPS plasmids \\
Lg-pR2 & ACCAACAGCGACAATAATGACG & CPS plasmids \\
Lg-Cps-1F & AGCAGCGTAGGCTATAGAAGCA & CPS plasmids \\
Lg-Cps-37R & TCTTGTCCCAGAGGGTTCTCCT & CPS plasmids \\
Lg-Cps-38R & ATGTTTCCCGTGTCCGTGCATC & CPS plasmids \\
Lg-Cps-38F & CCATTCCGACACGAAACTGATC & Chromosome (Lg2 type) \\
Lg-Cps-36R & ATCACTACGGTTGAGGATCATG & Chromosome (Lg2 type) \\
Lg-Cps-40F & GCTCCTGATGTAACCTATGCTTC & Chromosome (NUF462 type) \\
Lg-Cps-40R & CTGATGCTGCTGCATATGTTGAC & Chromosome (NUF462 type) \\
\hline
\end{tabular}

* The position of the primers was illustrated in Figs. 4, 5 and 6.

according to the manufacturer's instructions. The capsule synthetic gene locus of each strain was fragmentally amplified by PCR and sequenced as previously described (Kanai et al., 2017). The upstream and downstream regions of capsule gene cluster of each strain were amplified using an inverse PCR technique and sequenced as previously described (Kanai et al., 2017). The prediction of open reading frames and homology search on the newly found sequences were performed using DNASIS software (Hitachi software) and BLAST program at National Center for Biotechnology Information (https://blast.ncbi.nlm.nih.gov/Blast.cgi), respectively. ClustalW2.1 network version at DNA Data Bank of Japan (http://clustalw.ddbj.nig.ac.jp/index. php?lang) was used for DNA multiple sequence alignment.

The sequence data of the NUF462 chromosomal capsule gene cluster with franking region and CPS plasmids, pNUF18, pNUF462 and pNs15sousui29, are available on DDBJ/GenBank/EMBL databases under accession numbers LC324727, LC316979, LC316980 and LC316981, respectively.

Identification of CPS plasmids and integration site of chromosomal capsule gene cluster

The presence and differentiation of CPS plasmids in the $L$. garvieae strains were investigated by a PCR method using TaKaRa Ex Taq Polymerase (Takara Bio). Primer pairs used for amplification were Lg-pF2 / Lg-Cps37R and Lg-pF2/Lg-Cps-38R for the upper region of the capsule gene cluster and Lg-Cps-1F/Lg-pR2 for the lower region of the capsule gene cluster (Table 3 ). The
PCR reaction mixture $(50 \mu \mathrm{L})$ contained $5 \mu \mathrm{L}$ of $10 \times$ PCR buffer for TaKaRa Ex Taq Hot Start Version, 0.4 mM of each dNTP, $0.5 \mu \mathrm{m}$ of each primer, $1 \mu \mathrm{L}$ of template DNA and $1.25 \mathrm{U}$ of TaKaRa Ex Taq Hot Start Version. The PCR condition was as follows; initial denaturation at $95^{\circ} \mathrm{C}$ for $3 \mathrm{~min}$ and 30 cycles of denaturation at $98^{\circ} \mathrm{C}$ for $10 \mathrm{~s}$, annealing at $65^{\circ} \mathrm{C}$ for $30 \mathrm{~s}$ and extension at $72^{\circ} \mathrm{C}$ for $3 \mathrm{~min}$. The expected sizes of PCR products using the above-mentioned primer pairs were 3,325 and $1,296 \mathrm{bp}, 3,325$ and 1,296 bp, 4,330 and 2,301 bp and 4,330 and 2,301 bp for the upper region and 1,541, $2,737,1,541$ and 526 bp for the lower region of pNUF18, pNUF462, pBSLG13015 and pNs15sousii29, respectively.

The integration site of the chromosomal capsule gene cluster in the strains was investigated by a long PCR using KOD FX Neo Polymerase mentioned above. Primer pairs used were $\mathrm{Lg}-\mathrm{Cps}-38 \mathrm{~F} / \mathrm{Lg}-\mathrm{Cps}-36 \mathrm{R}$ for the Lg2 type integration site and Lg-Cps-40F/Lg-Cps-40R for the NUF462 type integration site (Table 3).

\section{Antimicrobial susceptibility test}

Susceptibility of the $L$. garvieae strains to erythromycin and lincomycin was studied using microplate-cultivation method. Briefly, fresh culture of each strain was inoculated into $150 \mu \mathrm{L}$ of Muller-Hinton broth supplemented with erythromycin $(25 \mu \mathrm{g} / \mathrm{mL})$ or lincomycin (5 $\mu \mathrm{g} / \mathrm{mL}$ ) in a well of a 96-well microplate and incubated for $24 \mathrm{~h}$ at $27^{\circ} \mathrm{C}$. Strains that grew in the wells were judged to be resistant to respective antibiotics. The bacterial growth in each well was assessed from turbidity measured at $600 \mathrm{~nm}$ with a microplate reader, Multi- 
skan FC (Thermo Scientific).

\section{Virulence test}

The two colony types of $L$. garvieae NUF462, opaque and relatively transparent, were cultured on $\mathrm{TH}$ agar for $24 \mathrm{~h}$. The bacterial suspensions were prepared in $0.01 \mathrm{M}$ phosphate buffered saline, pH 7.2 (PBS), serially 10 fold-diluted with PBS and intramuscularly injected to ten yellowtail per dilution at a rate of 0.1 $\mathrm{mL} / 100 \mathrm{~g}$ body weight. The control fish were injected with PBS. The average body weight of experimental fish was $57.6 \mathrm{~g}$. After inoculation, ten fish received the same dilution were stocked in a 100-L circular aquarium with constant water flow and reared for 3 wk with feeding. The average water temperature was $25.7^{\circ} \mathrm{C}$.

\section{Results}

Relationship between colony morphology and serological phenotype

When the stocked L. garvieae $\mathrm{KG}^{-}$strains were cultured on $\mathrm{TH}$ agar, two colony types, namely, opaque colony and relatively transparent one, grew from many strains like filefish isolates (Fig. 2). The results of slide

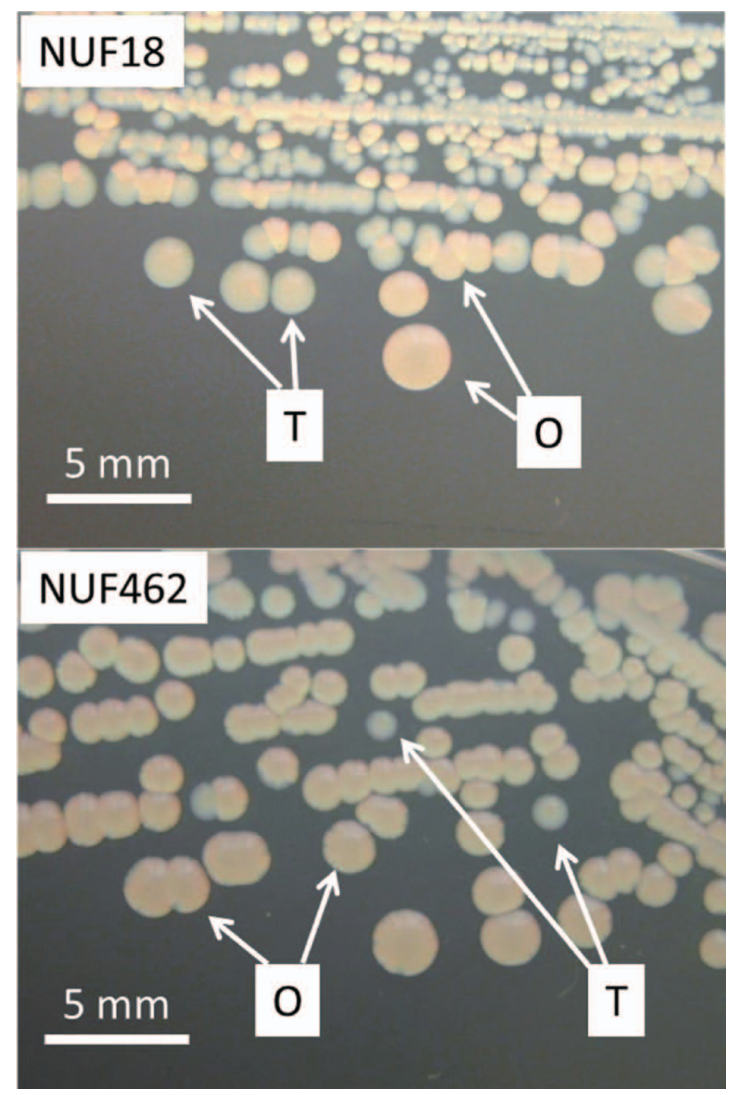

Fig. 2. Opaque $(\mathrm{O})$ and relatively transparent $(\mathrm{T})$ colonies of L. garvieae NUF18 (group 1) and NUF462 (group 2) grown on Todd Hewitt agar cultured for 2 days at $27^{\circ} \mathrm{C}$. The photographs were taken with a transmitted light that obliquely illuminated the agar plates. agglutination test revealed that the strains were divided into three groups. In one group opaque colonies were $\mathrm{KG}^{-}$type, while relatively transparent colonies were $\mathrm{KG}^{+}$ type (group 1). In the second group both colony types were $\mathrm{KG}^{-}$type (group 2). Strains that exhibited only one colony morphology also existed, and such strains were revealed to be $\mathrm{KG}^{-}$type (group 3).

Detection of capsule gene cluster in the genome of $\mathrm{L}$. garvieae strains

In the strains belonged to group 1, opaque colonies (KG- type) exhibited the plasmid capsule gene cluster, while relatively transparent colonies $\left(\mathrm{KG}^{+}\right.$type) did not. The chromosomal capsule gene cluster was not detected in either colony type (Fig. 3). On the other hand, in several strains belonged to group 2, opaque colonies ( $\mathrm{KG}^{-}$type) exhibited the plasmid capsule gene cluster, but relatively transparent colonies ( $\mathrm{KG}^{-}$type) exhibited neither the plasmid nor chromosomal capsule gene cluster. Then the integration site of the capsule gene cluster in the relatively transparent colonies of a representative strain, $L$. garvieae NUF462, was investigated, and it was revealed that the gene cluster was integrated in the chromosome at a position different from L. garvieae Lg2. Moreover, it was found that in the NUF462 opaque colonies the chromosomal gene cluster was detected at the same position as the relatively transparent colonies, that is, the opaque colonies of NUF462 was revealed to possess both the plasmid and chromosomal capsule gene clusters simultaneously (Fig. 3). In the rest of group 2 strains both opaque and relatively transparent colonies exhibited only the chromosomal capsule gene cluster at the same position as Lg2. In the $\mathrm{KG}^{-}$strains that exhibited only one colony morphology (group 3) the plasmid or chromosomal capsule gene cluster at the same position as NUF462 or Lg2 was detected.

The plasmid or chromosomal capsule gene cluster was detected in some $\mathrm{KG}^{+}$strains. In such cases, it is possible that mutation occurred in the capsule synthetic genes, but we did not elucidate this in this study.

\section{Structure of integration site of chromosomal capsule gene cluster}

The chromosomal capsule gene cluster of NUF462 was flanked by two IS982 elements like Lg2 and integrated in the spacer region between LCGL_1603 and LCGL_1604 of the Lg2 genome (GenBank accession no. NC_017490). A DNA sequence consisted of nine nucleotides, ACAATAAAA, which seemed to be a target sequence existed at both sides of the inserted capsule gene cluster plus IS982 elements (Fig. 4). In Lg2 and the other representative strains, NUF18, Ns12sousui23 and Ns15sousui29, this short sequence existed singly. Similarly, in the case of integration site in Lg2, a sequence consisted of AATATACAA was found at both 


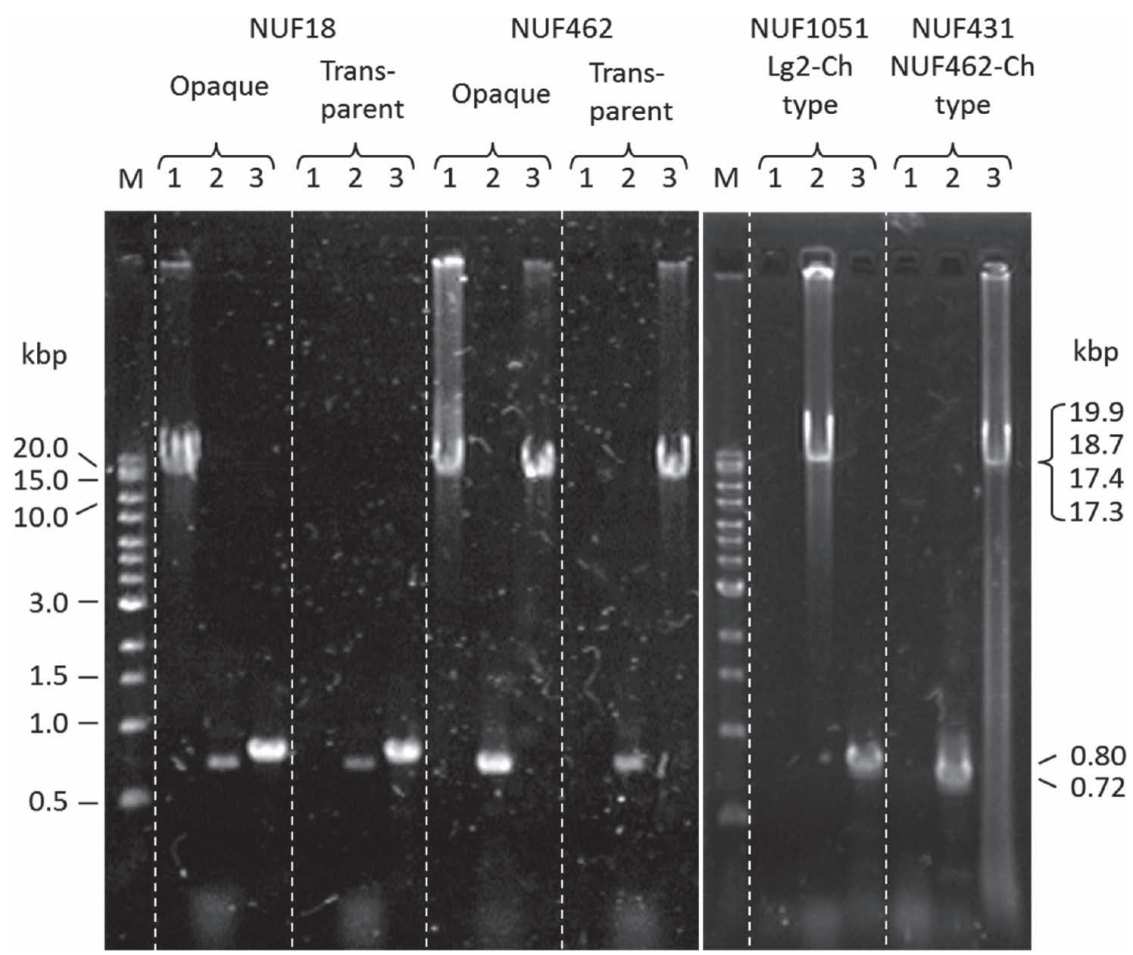

Fig. 3. Detection of plasmid and chromosomal capsule gene clusters in L. garvieae NUF18 opaque (KG ${ }^{-}$, pNUF18 type) and relatively transparent colonies (KG+ type), NUF462 opaque (KG', pNUF462 \& NUF462-Ch type) and relatively transparent colonies (KG', NUF462-Ch type), NUF1051 (KG', Lg2-Ch type) and NUF431 (KG', NUF462-Ch type) by PCR. 1, plasmid capsule gene cluster. Appearance of a band at 18.7 and $19.9 \mathrm{kbp}$ indicates the presence of the gene cluster (pNUF18 and pNUF462 type, respectively). 2, Lg2 type chromosomal capsule gene cluster (Lg2-Ch type). Appearance of bands at 17.3 $\mathrm{kbp}$ and $720 \mathrm{bp}$ indicates the presence and absence of the gene cluster, respectively. 3, NUF462 type chromosomal capsule gene cluster (NUF462-Ch type). Appearance of bands at $17.4 \mathrm{kbp}$ and $800 \mathrm{bp}$ indicates the presence and absence of the gene cluster, respectively. M, DNA size marker.

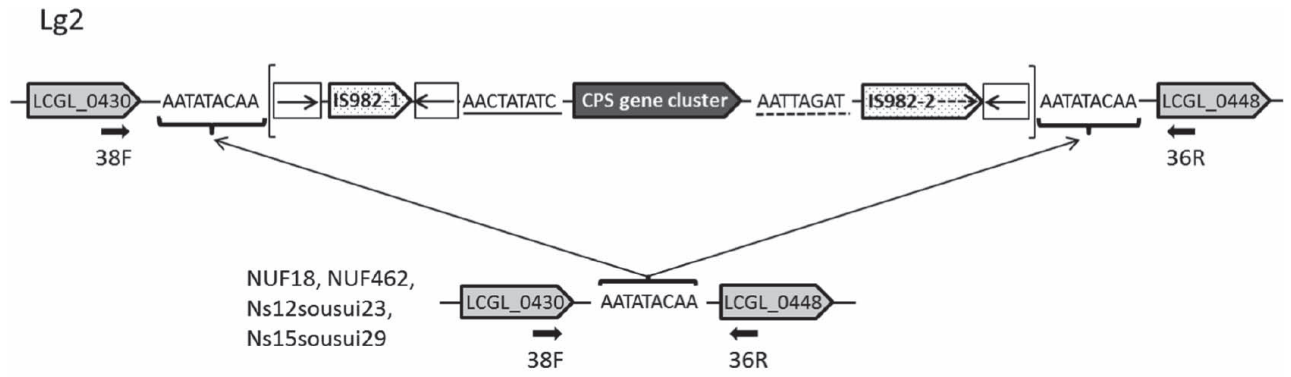

NUF462

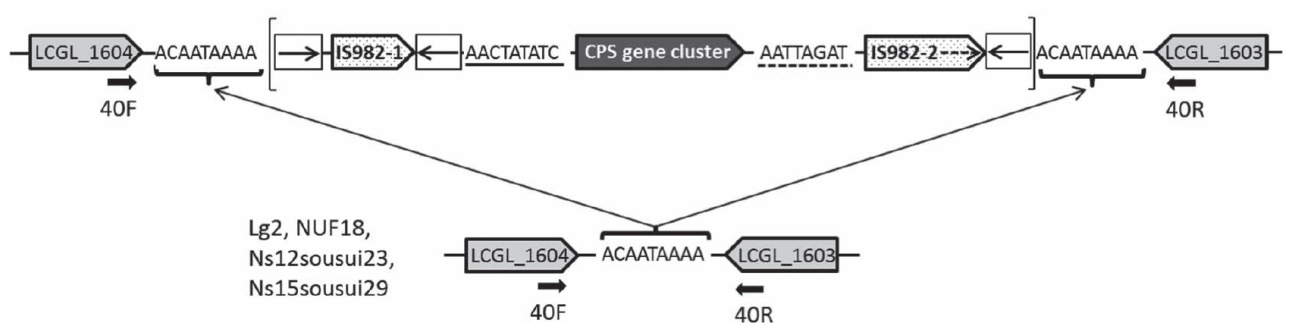

Fig. 4. Structure of the integration site of chromosomal capsule gene cluster in L. garvieae Lg2 and NUF462. Genes are not drawn to scale. The figure of Lg2 was drawn based on the sequence data of GenBank accession no. NC_017490. Sequence in brackets is a cassette structure also observed in CPS plasmids. Underlined sequences consisted of eight or nine nucleotides are target sequences detected in CPS plasmids (see Fig. 6). $\rightarrow$ and $\rightarrow$, inverted repeat sequences, which are also detected in CPS plasmids, (see Fig. 6). Short arrows indicate the position and direction of PCR primers used for detection and differentiation of the integration site of chromosomal capsule gene cluster: 38F, Lg-Cps-38F; 36R, Lg-Cps-36R; 40F, Lg-Cps-40F; 40R, Lg-Cps-40R. 

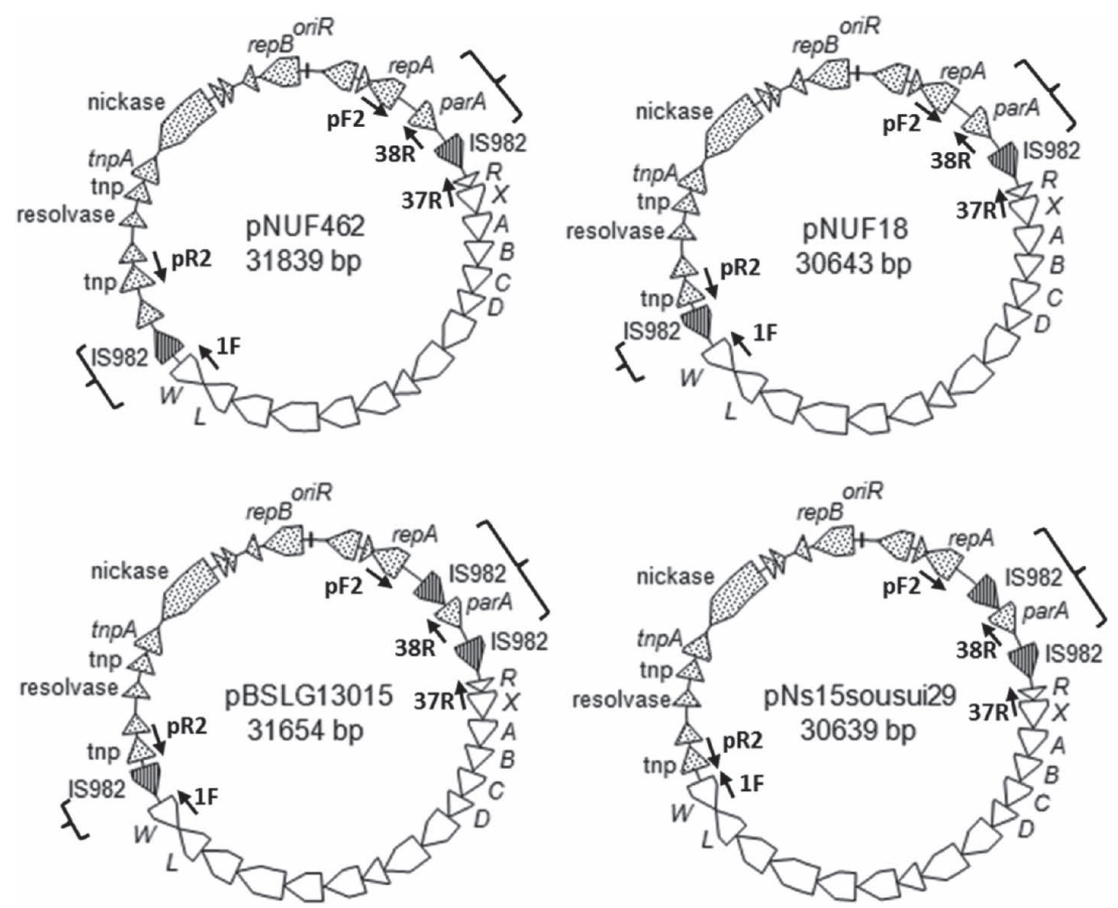

Fig. 5. Structure of the CPS plasmids pNUF462, pNUF18, pBSLG13015 (Kanai et al., 2017) and pNs15sousui29. Boxes with no pattern are the genes of capsule gene cluster. $R$ to $W$, epsR, epsX, eps $A$, epsB, epsC, epsD, eps $L$ and epsW. tnp, transposase. Arrows indicate the position and direction of PCR primers used for detection and differentiation of CPS plasmids: pF2, Lg-pF2; pR2, Lg-pR2; 1F, Lg-Cps-1F; 37R, Lg-Cps-37R; 38R, Lg-Cps-38R. Curly braces indicate the variable regions.

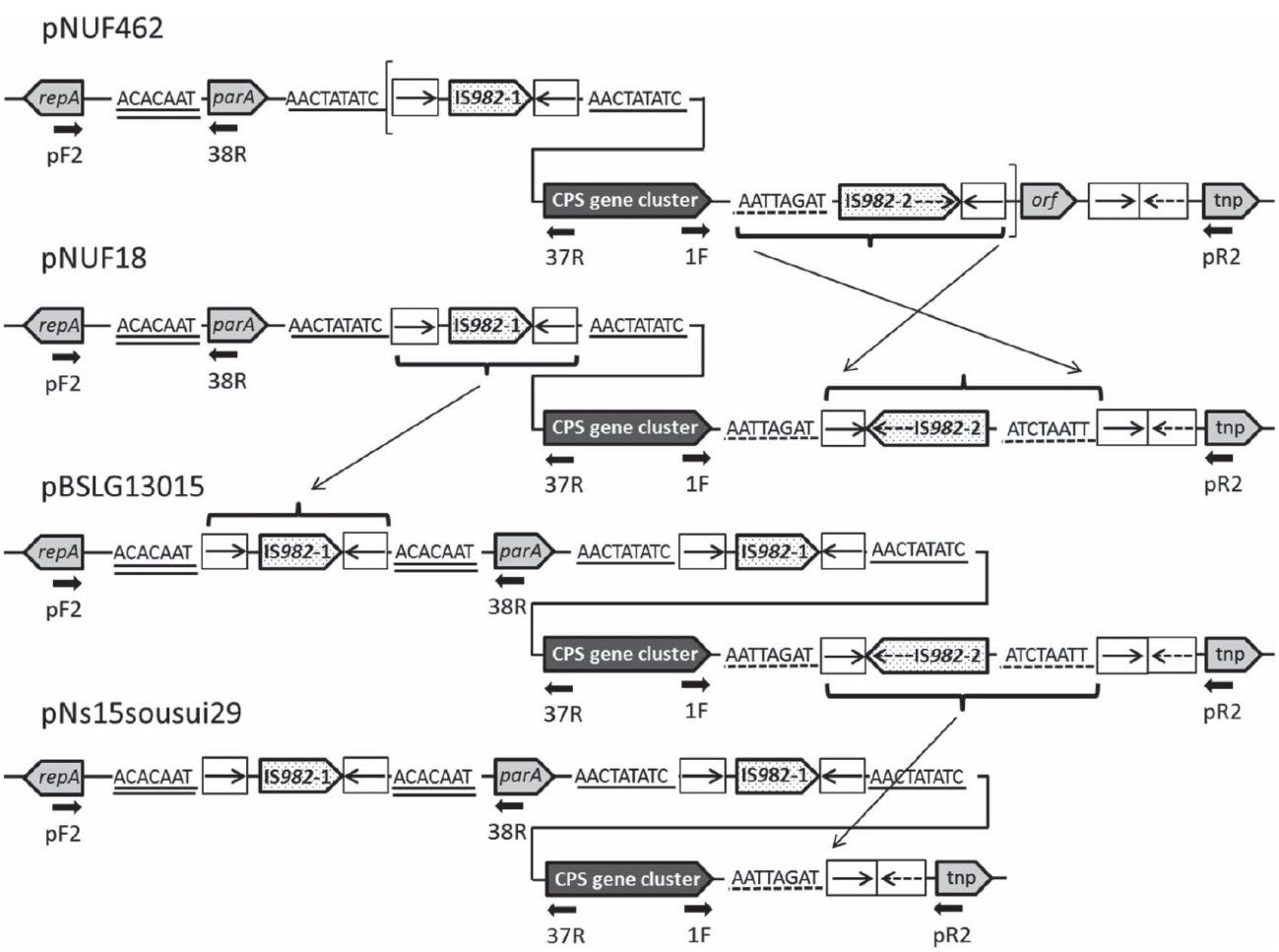

Fig. 6. Structure of upstream and downstream regions adjacent to the capsule gene cluster of CPS plasmids. Genes are not drawn to scale. Sequence in brackets is a cassette structure also observed in the chromosome. Underlined sequences consisted of seven to nine nucleotides are target sequences for IS982 element. $\rightarrow$ and $\rightarrow$, inverted repeat sequences, ACCCGAATTGCTAGTTGATT and TTCTATCGGGTATCAAGTCATGGCTAAAT, respectively. Short arrows indicate the position and direction of PCR primers used for detection and differentiation of CPS plasmids: pF2, Lg-pF2; pR2, Lg-pR2; 1F, Lg-Cps-1F; 37R, Lg-Cps-37R; 38R, Lg-Cps-38R. 
sides of the capsule gene cluster. Two kinds of inverted repeat sequences consisted of 20 and 29 nucleotides, respectively, were found adjacently to IS982 elements (Fig. 4).

\section{Structure of CPS plasmids}

Sequence analyses of plasmids from the four representative strains, NUF18, NUF462, Ns12sousui23 and Ns15sousui29, resulted in the discovery of three novel CPS plasmids from respective strains, designated pNUF18, pNUF462 and pNs15sousui29 (Fig. 5). The plasmid from Ns12sousui23 had the same structure as the CPS plasmid pBSLG13015 from filefish (Accession no. LC205736). The structure of these four plasmids resembled each other, but the upstream and downstream regions adjacent to the capsule gene cluster were different. The direction of two IS982 elements that sandwiched the capsule gene cluster in pNUF462 was the same as the chromosomal gene cluster of NUF462 and Lg2. In pNUF18 and pBSLG13015 the downstream IS982 was settled in the opposite direction compared to pNUF462. In pNs15sousui29 and pBSLG13015 another IS982 element was inserted between repA and parA genes. In Ns15sousui29 the downstream IS982 was lost. Like the integration sites of chromosomal capsule gene cluster, sequences consisted of seven to nine nucleotides were found to sandwich IS982 elements. The two kinds of repeat sequences consisted of 20 and 29 nucleotides were found to sandwich IS982 elements inversely or directly (Fig. 6).
Detection of CPS plasmids and integration site of chromosomal capsule gene cluster among the strains

The results of PCR for the detection and differentiation of the integration site of chromosomal capsule gene cluster and CPS plasmids are shown in Figs. 3 and 7. By the PCR the stocked $L$. garvieae strains were differentiated into eight categories (Fig. 8). Strains that possessed pNUF18 or pNUF462 (pNUF18 and pNUF462 type, respectively) were found till the year of 1991. Strains whose capsule gene cluster was integrated in the chromosome at the same position as NUF462 (NUF462-Ch type) were found between 1989 and 1992, most of which also possessed pNUF462 (pNUF462 \& NUF462-Ch type). Strains whose capsule gene cluster was integrated in the chromosome at the same position

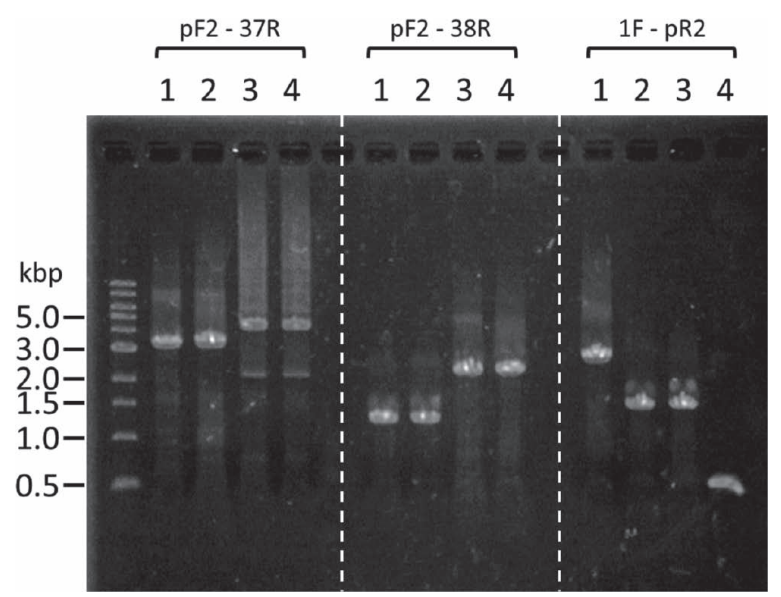

Fig. 7. Differentiation of CPS plasmids by PCR. 1, pNUF462; 2, pNUF18; 3, pBSLG13015; 4, pNs15sousui29. pF2, Lg-pF2; pR2, Lg-pR2; 1F, Lg-Cps-1F; 37R, Lg-Cps37R; 38R, Lg-Cps-38R

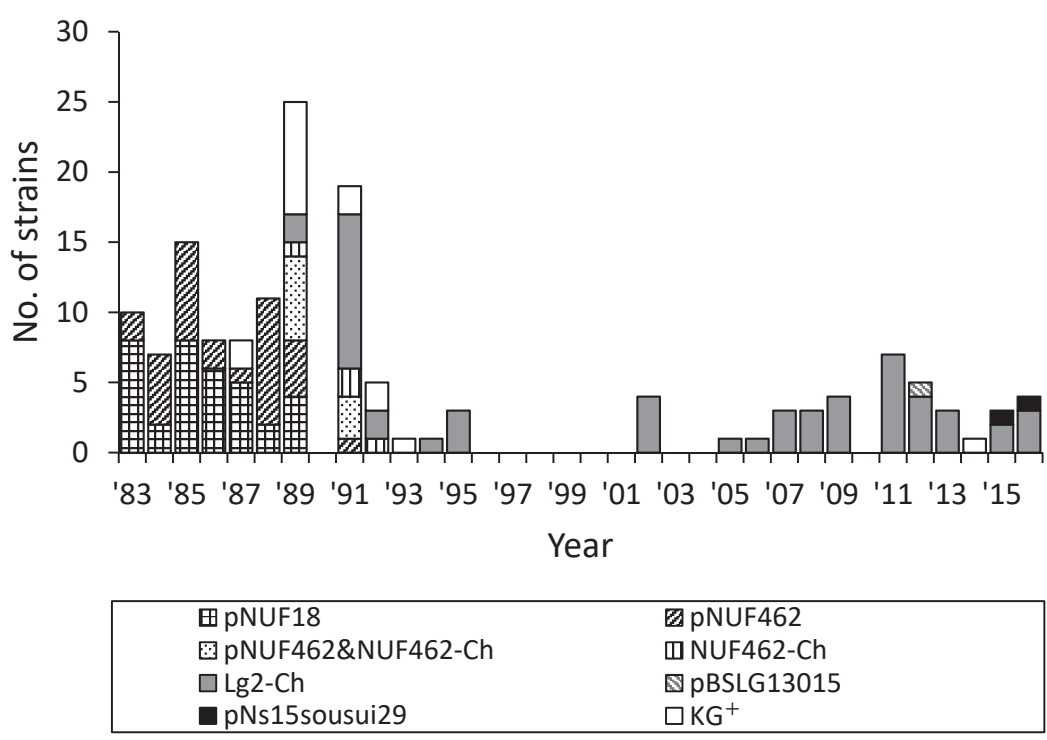

Fig. 8. Distribution of CPS plasmids (pNUF18, pNUF462, pBSLG13015, pNs15sousui29) and chromosomal capsule gene cluster (NUF462-Ch, Lg2-Ch) among L. garvieae strains. Neither CPS plasmids nor chromosomal capsule gene cluster were detected in the strains categorized as $\mathrm{KG}^{+}$. 
Table 4. Antibiotic susceptibility of the L. garvieae strains

\begin{tabular}{|c|c|c|c|c|c|}
\hline \multirow{3}{*}{$\begin{array}{l}\text { Integration site of } \\
\text { capsule gene cluster }\end{array}$} & \multirow{3}{*}{$\begin{array}{l}\text { Total no. } \\
\text { of strain }\end{array}$} & \multicolumn{4}{|c|}{ Susceptibility to: } \\
\hline & & \multicolumn{2}{|c|}{ Erythromycin } & \multicolumn{2}{|c|}{ Lincomycin } \\
\hline & & $S$ & $\mathrm{R}$ & $S$ & $\mathrm{R}$ \\
\hline pNUF18 & 35 & 35 & 0 & 35 & 0 \\
\hline pNUF462 & 31 & 30 & 1 & 30 & 1 \\
\hline pNUF462 \& NUF462-Ch & 9 & 2 & 7 & 2 & 7 \\
\hline NUF462-Ch & 4 & 1 & 3 & 1 & 3 \\
\hline \multicolumn{6}{|l|}{ Lg2-Ch } \\
\hline 1989-1994 & 16 & 16 & 0 & 10 & 6 \\
\hline $1995-2016$ & 38 & 38 & 0 & 1 & 37 \\
\hline pBSLG13015 & 1 & 1 & 0 & 1 & 0 \\
\hline pNs15sousui29 & 2 & 2 & 0 & 2 & 0 \\
\hline
\end{tabular}

$\mathrm{S}$, susceptible; R, resistant; Ch, chromosome.

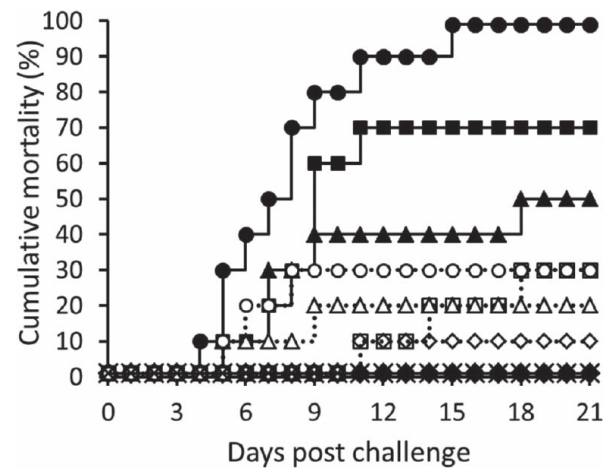

Fig. 9. Virulence of opaque $\left(\boldsymbol{O}, \boldsymbol{\Delta}, \boldsymbol{\square}, \boldsymbol{\nabla} ; 1.6 \times 10^{4}-10^{1}\right.$ CFU/100 g body weight, respectively) and relatively transparent colonies $\left(\bigcirc, \triangle, \square, \diamond ; 1.6 \times 10^{4}-10^{1}\right.$ CFU/100 g body weight, respectively) of $L$. garvieae NUF462 to yellowtail studied by intramuscular inoculation. The control fish $(x)$ were injected with PBS.

as Lg2 (Lg2-Ch type) were found between 1989 and 2016. pBSLG13015 was detected only in Ns12sousui23 isolated from Pacific bluefin tuna, and pNs15sousui29 was detected in two strains isolated from kingfish $S$. lalandi in 2015 (Ns15sousui29) and 2016, but the other strains derived from these fish species belonged to Lg2Ch type. Strains categorized as $\mathrm{KG}^{+}$, in which neither CPS plasmids nor chromosomal capsule gene cluster were detected, were supposed to be $\mathrm{KG}^{+}$derivatives of CPS plasmid type (Fig. 8).

Relationship between integration site of capsule gene cluster and antibiotic susceptibility

As shown in Table 4, almost all the strains of pNUF18 and pNUF462 types were susceptible to erythromycin and lincomycin, but the majority of strains of NUF462-Ch and pNUF462 \& NUF462-Ch types were resistant to both antibiotics. In Lg2-Ch type one-third of strains isolated between 1989 and 1994 were resistant to lincomycin, and almost all the strains isolated between 1995 and 2016 were resistant to this antibiotic. Strains possessed pBSLG13015 and pNs15sousui29 were susceptible to both antibiotics.
Virulence of opaque and relatively transparent colonies of NUF462 to yellowtail

The mortalities in the fish groups inoculated with the opaque colonies from $10^{4}$ to $10^{2} \mathrm{CFU} / 100 \mathrm{~g}$ body weight were higher than those inoculated with the relatively transparent colonies (Fig. 9). The result indicates that the opaque colonies of NUF462 are more virulent than the relatively transparent ones.

\section{Discussion}

In this study we have shown that the integration site of the capsule gene cluster of $L$. garvieae was varied among the strains tested. Some strains possessed one of the four CPS plasmids, and the others possessed the capsule gene cluster in the chromosome at the same position as L. garvieae NUF462 or Lg2. Moreover, some strains simultaneously possessed both the CPS plasmid and chromosomal capsule gene cluster. In $L$. lactis several kinds of gene cluster for exopolysaccharide production have been found, and their integration sites differ according to gene cluster, namely in plasmids or chromosome (Morita et al., 2011). In L. garvieae the capsule gene cluster in the plasmids and chromosome was the same, suggesting that the structure and serological properties of the capsular polysaccharide produced are the same despite that the integration sites are different. Previously we reported that all the L. garvieae strains isolated from filefish possessed the CPS plasmid pBSLG13015 and thought that the CPS plasmid was restricted to filefish isolates (Kanai et al., 2017). However, from the present study it is certain that CPS plasmids are widely distributed among the Japanese $L$. garvieae isolates not only from filefish but from the other fish species.

There seems to be a transition of the integration site of the capsule gene cluster. In this study pNUF18 and pNUF462 were detected in the strains isolated between 1983 and 1991. Their serological phenotypic change from $\mathrm{KG}^{-}$to $\mathrm{KG}^{+}$type occurred by subculturing probably due to plasmid curing. Since the phenotypic change 
from $\mathrm{KG}^{-}$to $\mathrm{KG}^{+}$is a well-known character of $L$. garvieae (Kitao, 1982), the majority of $L$. garvieae isolated before 1983 are also supposed to be the plasmid type. The strains of NUF462-Ch and Lg2-Ch types appeared in 1989, and the Lg2-Ch type prevailed till 2016. Their serological phenotypic change seemed to occur at a relatively low frequency compared to the strains possessing CPS plasmids (Fig. 1). A previous paper reported the frameshift mutations existed in the capsule synthetic genes of $L$. garvieae Lg2-S, a KG derivative of Lg2 (Morita et al., 2011). In the present study not a few $\mathrm{KG}^{+}$ strains belonged to Lg2-Ch type were found (data not shown). So, in cases of the $L$. garvieae isolates possessing the chromosomal capsule gene cluster the phenotypic change may occur due to mutation in the capsule synthetic genes rather than loss of the gene cluster.

The four $L$. garvieae CPS plasmids described in this paper had similar structure, but the upstream and downstream regions adjacent to the capsule gene cluster were varied among them. The comparison of the variable regions indicate that the transition of structure in the order of pNUF462 $\rightarrow$ pNUF18 $\rightarrow$ pBSLG13015 $\rightarrow$ pNs15 sousui29 might have occurred by inversion, replication and deletion of the IS982 element. It is possible that the chromosomal capsule gene cluster may be translocated from pNUF462, because 1) the structure of a cassette consisted of capsule gene cluster plus two IS982 elements in the chromosome is the same as that of pNUF462, 2) the earlier L. garvieae strains tended to possess the CPS plasmids only, 3) both pNUF462 and the chromosomal capsule gene cluster co-existed in some strains, and 4) the repeat sequence consisted of 20 nucleotides was situated inversely at the both ends of the cassette structure like as IS982 element (Fig. 4). It is known that the inverted repeat sequences of ISs act as a receptor for transposase, so they are essential for the transposition of IS elements (Szabō et al., 2010).

It is interesting that the susceptibility of the $L$. garvieae strains to erythromycin and lincomycin was related to the integration site of the capsule gene cluster. Multiple drug resistant $L$. garvieae including macrolide resistance emerged and prevailed at yellowtail aquaculture sites around 1990, and, recently, lincomycin-resistant $L$. garvieae isolates have increased (Aoki et al., 1990; Nishiki et al., 2011; Furushita et al., 2015). It is possible that the integration site might have influenced the acquisition of resistance, or, conversely, the acquisition of resistance might have affected the change in the integration site. To solve this question a further study will be needed by increasing the number of $L$. garvieae isolates examined.

In this study the two colony morphologies, opaque and relatively transparent, were observed among the $L$. garvieae strains as filefish isolates (Kanai et al., 2017). These colony morphologies probably represent encapsulated or non-encapsulated, respectively, in the strains possessing the CPS plasmid only such as NUF18 and filefish isolates. In this case the colony opacity may reflect encapsulation. On the other hand, in the strains such as NUF462, although the two colony types were observed, both the types were $\mathrm{KG}^{-}$phenotype, namely encapsulated. It is possible that the colonies possessing both the CPS plasmid and chromosomal capsule gene cluster may produce capsular polysaccharide more abundantly and become opaquer than the colonies possessing the chromosomal gene cluster only. The abundance of capsular polysaccharide may be related to the virulence. In the present study opaque colonies of NUF462 were revealed to be more virulent than transparent ones. If the copy number of CPS plasmids in a bacterial cell is more than two, the amount of capsular polysaccharide produced may increase. In a previous study we have shown that the virulence of a $L$. garvieae strain isolated from filefish which possesses the CPS plasmid pBSLG13015 was higher than that isolated from yellowtail, whose capsule gene cluster is integrated in the chromosome only (Kanai et al., 2017). In conclusion, it is suggested that $L$. garvieae isolates possessing CPS plasmids form opaque colonies and are highly virulent compared to those possessing the chromosomal capsule gene cluster only.

\section{References}

Aoki, T., K. Takami and T. Kitao (1990): Drug resistance in a non-hemolytic Streptococcus sp. isolated from cultured yellowtail Seriola quinqueradiata. Dis. Aquat. Organ., 8, 171-177.

Furushita, M., T. Fukuda, Y. Fukuda, A. Yamashita, S. Yanagi, Y. Imaoka, S. Tanaka, Y. Sugihara, M. Abe, T. Nagano, S. Aono, H. Miyazawa and T. Shiba (2015): Antimicrobial susceptibility of the strains of Lactococcus garvieae isolated from cultured fish of the genus Seriola (yellowtail, amberjack and kingfish) in Japan from 2004 to 2009. Aquacult. Sci., 63, 59-64. (in Japanese with English summary)

Kanai, K., M. Tsujikura, K. Shutou, T. Honma, F. Matsumoto, K. Suga, S. Takagi, Y. Fukuda and Y. Sugihara (2017): A novel plasmid carrying capsule gene cluster found in Lactococcus garvieae isolated from filefish. Fish Pathol., 52, 82-88.

Kawanishi, M., T. Yoshida, S. Yagashiro, M. Kijima, K. Yagyu, T. Nakai, M. Murakami, H. Morita and S. Suzuki (2006): Differences between Lactococcus garvieae isolated from the genus Seriola in Japan and those isolated from other animals (trout, terrestrial animals from Europe) with regard to pathogenicity, phage susceptibility and genetic characterization. J. Appl. Microbiol., 101, 496-504.

Kitao, T. (1982): The methods for detection of Streptococcus sp. causative bacteria of streptococcal disease of cultured yellowtail (Seriola quinqueradiata). Fish Pathol., 17, 17-26. (in Japanese with English summary)

Minami, T., M. Kanemaru, K. Iwata, K. Nakanishi, A. Yamashita, Y. Miyoshi, Y. Fukuda and T. Yoshida (2012): Occurrences of diseases in thread-sail file fish in western part of Japan. Fish Pathol., 47, 111-113. (in Japanese with English summary)

Miyauchi, E., H. Toh, A. Nakano, S. Tanabe and H. Morita 
(2012): Comparative genomic analysis of Lactococcus garvieae strains isolated from different sources reveals candidate virulence genes. Int. J. Microbiol., 2012, article ID 728276, doi: 10.1155/2012/728276.

Morita, H., H. Toh, K. Oshima, M. Yoshizaki, M. Kawanishi, K. Nakaya, T. Suzuki, E. Miyauchi, Y. Ishii, S. Tanabe, M. Murakami and M. Hattori (2011): Complete genome sequence and comparative analysis of the fish pathogen Lactococcus garvieae. PLoS One, 6, e23184.

Nishiki, I., M. Furukawa, S. Matui, T. Itami, T. Nakai and T. Yoshida (2011): Epidemiological study on Lactococcus garvieae isolates from fish in Japan. Fish. Sci., 77, 367-
373.

Szabō, M., J. Kiss and F. Olasz (2010): Functional organization of the inverted repeats of IS30. J. Bacteriol., 192, 34143423.

Yoshida, T. (2016): Streptococcosis in aquaculture. Fish Pathol., 51, 44-48. (in Japanese with English summary)

Yoshida, T., M. Endo, M. Sakai and V. Inglis (1997): A cell capsule with possible involvement in resistance to opsonophagocytosis in Enterococcus seriolicida isolated from yellowtail Seriola quinqueradiata. Dis. Aquat. Organ., 29, 233-235. 